\title{
Le(s) long(s) parcours de la mise sur le marché des médicaments
}

Christophe Duguet

\begin{abstract}
$>$ Entre le premier essai clinique d'un médicament en développement et sa commercialisation, la route est longue et semée d'embûches. De raccourcis en itinéraire bis, d'évaluations rendues publiques en négociations sécrètes, ce dédale réglementaire paraît aussi hermétique que rébarbatif au néophyte. Il a pourtant des impacts très concrets sur l'accès des malades aux thérapies innovantes. <
\end{abstract}

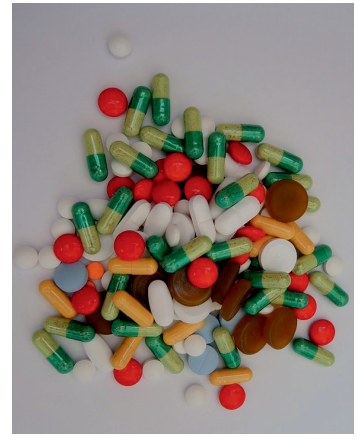

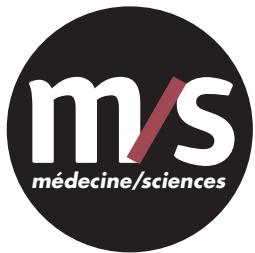

médecine/sciences $2019 ; 35$ (hors série $n^{\circ} 1$ ) : 13-7
Directeur des affaires publiques, AFM-Téléthon, 1 , rue de l'internationale, 91002 Évry, France. cduguet@afm-telethon.fr
L'arrivée sur le marché d'un médicament innovant ne relève pas d'un effet off/on. II ne s'agit pas d'une bascule instantanée entre un avant sans, et un après avec. C'est au contraire un processus très progressif et qui prend du temps. Le chemin qui conduit à la commercialisation se décline au pluriel. II n'existe pas un parcours réglementaire, mais des parcours réglementaires. Ils partagent un certain nombre d'étapes, obligatoires, mais se différencient par des chemins de traverse liés aux spécificités de chaque principe actif. Dans tous les cas, la route est longue (voire très longue) et tortueuse en raison de la complexité d'une réglementation qui, de surcroît, évolue en permanence. Des nouveautés réglementaires sont déjà annoncées pour les mois et même les semaines à venir. Il faut en permanence se tenir à jour.

\section{Une succession d'incontournables}

En Europe, le parcours réglementaire d'un médicament innovant, notamment dans le domaine des maladies rares, commence souvent avant même les essais cliniques. II s'agit de la demande et de l'obtention, par le laboratoire, de la désignation du statut de «médicament orphelin » au niveau européen. Ce statut donne quelques avantages, en termes de simplification, pour le développement. Si seule une petite partie des produits désignés « médicament orphelin » iront jusqu'à obtenir une autorisation de mise sur le marché (AMM), ils bénéficieront alors d'une protection relative puisque l'Union européenne s'engage dès lors à ne pas accepter, pendant une période de 10 ans, de nouveaux médicaments qui ne feraient pas mieux que le médicament déjà autorisé. La deuxième étape du parcours est celle des essais cliniques et donc, en amont, des demandes d'autorisations d'essais cliniques. Pour accorder cette autorisation, les autorités de santé jugent si, d'un point de vue réglementaire, elles autorisent des patients dûment informés à prendre un «risque » lié au nouveau traitement, au vue de premières données qui présument de son effet positif éventuel.
L'étape suivante consiste à préparer, en interaction avec les autorités, l'obtention de l'AMM. Cette autorisation de commercialisation est délivrée sur le constat que le médicament fait plus de bien que de mal. En d'autres termes, son rapport bénéfice/risque est positif. Néanmoins, la délivrance d'une AMM ne préjuge pas de la valeur du nouveau médicament dans la stratégie thérapeutique, par rapport aux autres produits déjà commercialisés. Cette appréciation relève d'une autre étape, appelée en anglais HTA, pour health technology assessment. Ses résultats nourrissent l'étape suivante, de fixation du niveau de prise en charge financière par la collectivité (taux de remboursement) puis de négociation du prix. La dernière étape, à ne pas oublier, est dite de «post-AMM »: une fois le prix fixé et le médicament en vente, des études complémentaires peuvent être demandées. Pour pratiquement tous les médicaments, il existe à ce stade des obligations en termes de registre et de suivi. Cette phase de post-AMM porte notamment des enjeux de pharmacovigilance.

\section{Des points communs européens et des spécificités nationales}

Les étapes successives du parcours se déroulent à différents niveaux territoriaux. La désignation de médicament orphelin et I'AMM relèvent par exemple d'une décision centralisée, à l'échelle de l'Europe. Les médicaments innovants pour nos maladies neuromusculaires sont soumis obligatoirement à une procédure gérée par l'Agence européenne du médicament (European Medicines Agency ou EMA). Une décision de mise sur le 
marché prise par l'EMA s'impose à l'ensemble du territoire européen. Concrètement, cela signifie que, pour la France, l'Agence nationale de sécurité du médicament et des produits de santé (ANSM) ne prend pas de décision d'AMM pour les médicaments innovants. II faut garder ce point important à l'esprit. A contrario, c'est à chaque pays de décider s'il autorise ou non un essai clinique à se dérouler sur son territoire. Dans l'Hexagone, cette décision revient à I'ANSM et à des comités d'éthique (les comités de protection des personnes ou CPP). Cette procédure va néanmoins changer très bientôt, sous l'impulsion d'un règlement européen qui date de quelques années mais n'est pas encore entré en application. À partir de 2020, la demande d'autorisation d'un essai clinique, qu'il concerne un seul pays ou plusieurs, relèvera d'une procédure unique centralisée à l'échelle européenne. En matière d'accès anticipé d'un médicament innovant au marché, chaque pays possède ses propres règles. Cet accès anticipé constitue un « raccourci » du parcours réglementaire-type, primordial dans les maladies rares pour lesquelles il n'existe pas d'alternative thérapeutique. Il peut s'ouvrir avant même l'obtention de l'AMM, sur la base de premiers résultats d'essais cliniques. Si des résultats permettent de penser qu'il y a une forte présomption que le médicament présente un réel intérêt pour soigner des patients, il peut y avoir un accès anticipé. Des patients peuvent ainsi en bénéficier avant que les différentes phases obligatoires (AMM, évaluation, négociation de prix...) n'aient abouti. La France a opté en ce domaine pour le système des autorisations temporaires d'utilisation (ATU) (Figure I) et des post-ATU, qui fait beaucoup d'envieux dans les autres pays. Les étapes d'évaluation HTA (health technology assessment), de négociation du prix et de prise en charge, relèvent de la responsabilité exclusive de chacun des États européens. Or ils adoptent des logiques, des approches et des procédures différentes. Le coût et le remboursement d'un même médicament peuvent donc s'avérer très différents d'un pays à l'autre. Bien que bénéficiant d'une AMM généralisée à l'ensemble de l'Union européenne, il peut ne pas être disponible dans un grand nombre de pays. En France, l'évaluation HTA revient à la Commission de la transparence et à la Commission évaluation économique et de santé publique (CE\&SP) de la Haute autorité de santé (HAS), la négociation du prix et la fixation du taux de remboursement au Comité économique des produits de santé (CEPS). Toutes ces décisions ne s'appliquent que pendant une durée limitée. En post-AMM sont opérées des révisions régulières aussi bien de l'AMM que de l'évaluation HTA du médicament. Les conclusions de ces réévaluations peuvent avoir des conséquences notamment sur le prix et le taux de remboursement du médicament, et éventuellement sur son maintien sur le marché. Les processus de mise sur le marché des médicaments innovants sont très longs. Entre l'obtention de l'AMM et la décision de prix, il ne devrait (en théorie) pas s'écouler plus de 180 jours. Et la moitié de cette durée réglementaire (90 jours) devrait être consacrée à l'évaluation HTA. En France, la HAS parvient peu ou prou à tenir ce délai. En revanche, les négociations de prix durent souvent beaucoup plus longtemps que les 90 jours restant prévus. Par exemple, des négociations de prix en cours pour certains médicaments indiqués dans des maladies rares ont débuté il y a 18 mois, voire deux ans ! Notre chance en France, c'est de pou- voir recourir au dispositif des ATU et de post-ATU. Ils permettent aux patients d'accéder à un médicament innovant avant que ces discussions n'aboutissent.

\section{Des paramètres médicaux et économiques}

L'évaluation de la HAS porte sur deux critères : le service médical rendu (SMR) et l'amélioration du service médical rendu (ASMR) (Figure 2). Déterminer le premier permet de répondre à la question : le médicament doitil être remboursé ? Pour le second, l'interrogation est différente : le produit améliore-t-il la situation clinique des patients par rapport aux autres traitements disponibles? Il s'agit donc d'un indicateur comparatif, relatif et non absolu. Pour déterminer le SMR d'un nouveau médicament, la Commission de la transparence prend en compte notamment les caractéristiques de la maladie (sévérité), l'efficacité et les effets indésirables du médicament, sa place dans la stratégie thérapeutique, son caractère préventif, curatif ou symptomatique et son intérêt pour la santé publique. L'analyse de ces critères permet d'attribuer un SMR important, modéré, faible ou insuffisant. À chacun correspond un taux de remboursement. Si le SMR est jugé insuffisant, il n'y aura pas de remboursement. On ne discute même plus du prix et dans la majorité des cas il ne sera pas réellement commercialisé. Le taux de remboursement sera au contraire de $65 \%$ pour un SMR important, $30 \%$ s'il est modéré et $15 \%$ s'il est faible. Cette dégressivité n'a finalement pas vraiment d'impact pour les maladies qui nous concernent puisqu'elles ouvrent droit à une prise en charge à $100 \%$ dans le cadre des affections longue durée (ALD). L'ASMR est pour sa part cotée de I (amélioration majeure du service médical rendu) à $V$ (pas d'amélioration). Moins d'un médicament par an se voit attribuer une ASMR I. La plupart obtiennent une ASMR IV ou V. Cette cotation a des conséquences en termes de négociation du prix. Pour une ASMR IV par exemple, le prix pourra être égal à celui des médicaments déjà disponibles auquel le nouveau médicament a été comparé. Pour une ASMR de I à III, son prix pourra être supérieur. Par ailleurs, un médicament avec une ASMR I, II ou III, et de façon exceptionnelle IV, pourra être inscrit sur la « liste en sus ». Ce système dérogatoire permet aux établissements de santé de financer des traitements excessivement coûteux. En principe, un établissement est remboursé par la Sécurité sociale de la prise en charge (notamment médicamenteuse) d'un patient via un paiement forfaitaire global. C'est le principe de la tarification à l'activité, la T2A. Or ces forfaits sont très insuffisants pour des médicaments innovants qui, trop souvent, sont excessivement onéreux. L'inscription de 


\section{Le cas particulier des molécules repositionnées}

Un médicament qui a déjà obtenu une AMM dans une indication peut s'avérer présenter un intérêt thérapeutique dans une autre pathologie. Pour cette nouvelle indication, si une extension d'AMM n'est pas demandée ou pas encore obtenue, la prescription peut se faire « hors AMM ». En effet, si la loi stipule que la prescription d'une spécialité pharmaceutique doit toujours être conforme à son AMM ou à son ATU, elle supporte une dérogation : un médecin peut prescrire en dehors de son AMM (indication thérapeutique, posologie, âge) un médicament disponible en France au vu des données acquises de la science, et sous sa responsabilité. Deux obligations s'imposent toutefois au prescripteur. La première est d'en informer le malade. La seconde est d'indiquer sur l'ordonnance que la prescription est réalisée hors AMM, une exigence de fait très rarement respectée... ce qui permet d'ailleurs au malade de se faire rembourser. Dans le domaine des maladies rares, l'Assurance maladie n'a de toute façon que rarement les moyens de savoir si la molécule a été prescrite hors AMM ou pas... Mais afin de sécuriser I'utilisation et le remboursement d'un médicament utilisé hors AMM, un dernier dispositif peut être sollicité. II s'agit de la recommandation temporaire d'utilisation (RTU). Comparable à une ATU de cohorte, la RTU est également délivrée par l'Agence nationale de sécurité des médicaments et des produits de santé à la demande, par exemple, d'un Centre de référence maladies rares. Néanmoins, ce dispositif réglementaire fonctionne mal et s'avère peu adapté à des nombreuses situations dans le domaine des maladies rares. II va probablement connaitre des évolutions qui pourraient faciliter son utilisation. ces médicaments particulièrement coûteux sur la « liste en sus » ouvre droit pour l'hôpital à un remboursement intégral de chaque boîte qu'il utilise. Par ailleurs, les médicaments dépassant un certain chiffre d'affaires font également l'objet d'une autre évaluation, menée cette fois par la Commission d'évaluation économique et de santé publique (CEESP) de la HAS. Elle porte sur l'efficience médico-économique des produits les plus innovants et les plus coûteux, en pratique ceux pour lesquels sont revendiqués par les industriels une ASMR I, II ou III et qui sont susceptibles d'avoir un «impact significatif » sur les dépenses de l'Assurance maladie. La CEદSP juge notamment de l'impact budgétaire global de l'arrivée du produit. Elle détermine également un paramètre assez complexe : le ratio différentiel coût-résultat (RDCR), qui évalue le coût supplémentaire en euros par année de vie gagnée en bonne santé (quality adjusted life year ou (aly). Son avis d'efficience éclaire les négociations de prix, sans pour autant fixer un seuil absolu.

\section{Un accès anticipé via l'ATU}

En amont, avant même l'obtention d'une AMM, un médicament innovant peut bénéficier d'une autorisation temporaire d'utilisation ou ATU. Géré par l'ANSM, ce possible accès anticipé au marché ne concerne que les produits destinés à traiter, prévenir ou diagnostiquer des maladies graves ou rares. II ne doit pas exister de traitement approprié déjà disponible sur le marché français. La présomption d’un rapport efficacité/sécurité positif du nouveau médicament doit être élevée. Ce traitement ne peut pas être différé sans risque d'une perte de chance importante pour les patients. Deux dispositifs coexistent, I'ATU nominative (ATUn) et l'ATU de cohorte (ATUc). La première concerne les situations où une autorisation d'utilisation anticipée est demandée pour un patient donné, par un médecin et sous sa responsabilité. Elle s'applique uniquement au cas où cette personne ne peut pas être incluse dans un essai clinique en cours et qu'il n'existe pas d'ATU de cohorte déjà en vigueur. Le dispositif de l'ATU nominative a été allégé en septembre 2018 avec la mise en place notamment d'une demande simplifiée si le médicament figure dans un référentiel ${ }^{1}$. Ce dernier recense près de 170 produits disponibles en ATU nominatives et précise, pour chacun d'entre eux, les critères d'octroi de l'autorisation d'usage anticipé et notamment les indications à respecter. A partir du moment où le médecin demandeur s'engage à se conformer à ces critères, il n'a pas à justifier sa demande et obtient rapidement l'ATU nominative. Sensiblement différente, l'ATU de cohorte est obtenue pour un groupe de patients à condition que le laboratoire s'engage à déposer sa demande d'AMM dans un délai fixé. Une fois l'ATUc en vigueur, elle s'applique à tous les patients qui répondent à des critères médicaux précis sans que le clinicien n'ait à justifier sa prescription pour chacun de ces patients. Ces critères sont définis dans un protocole d'utilisation thérapeutique (PTU) et de recueil d'informations.

\section{Des dispositions financières essentielles}

Toutes les ATU sont supprimées, de façon automatique, dans un délai de 1 à 2 mois une fois l'AMM obtenue et ce quelles que soient les indications qu'elle retient. En France, un relais a été créé afin de couvrir cette phase critique de suppression de l'accès anticipé : le dispositif post-ATU. II permet aux patients de continuer à bénéficier du nouveau médicament, alors même que son prix n'est pas encore fixé. Lorsque le champ des indications de l'AMM est plus large que celui de I'ATUc, les indications supplémentaires (ne figurant pas dans l'ATUc) peuvent tout de même bénéficier d'une prise en charge dans le cadre du dispositif post-ATU, à la condition toutefois qu'il n'existe pas d'alternative thérapeutique constatée par la Haute autorité de santé. Point important, pendant toutes les formes d'accès anticipé au marché (ATUn, ATUc, post-ATU), le laboratoire possède une liberté sur le

Référentiel des spécialités délivrées dans le cadre des ATU. 
prix de son médicament. II peut le facturer aux établissements de santé ou le leur délivrer gratuitement. Et lorsque le laboratoire choisit de le faire payer, c'est lui qui décide du montant de la facture. Cependant, une fois le prix définitif fixé par les autorités de santé, plusieurs mois ou années plus tard, le «trop perçu » éventuel du laboratoire en phases d'ATU et de post-ATU devra être remboursé aux établissements de santé. Dans tous les cas, et contrairement à une idée reçue, quel que soit le prix facturé par le laboratoire pendant la période des ATU et post-ATU, les hôpitaux se font rembourser intégralement leurs dépenses par l'Assurance maladie. Et il n'y a ni ligne budgétaire spécifique, ni plafonnement. Le coût d'un traitement en accès anticipé ne pèse donc pas sur le budget de l'hôpital. Il peut avoir à payer, parfois très cher, un médicament mais s'en fait rembourser chaque boite à $100 \%$. Cette disposition règlementaire doit balayer les réticences de certains hôpitaux, qui disent ne pas pouvoir dispenser de médicaments en ATU ou en post-ATU en raison de leurs coûts prohibitifs... Le seul obstacle, réel, à leur achat est celui de difficultés à faire une avance de trésorerie, une situation rare. Au bilan, l'achat d'un médicament en ATU ou en post-ATU ne coûte rien à un établissement de santé.

\section{Du prix officiel du produit, au coût réel du traitement}

La phase de négociation du prix entre le laboratoire et l'État ne concerne que les médicaments très coûteux ou ceux qui seront remboursés en ville. Les produits utilisés à l'hôpital ne sont pas concernés. Ils font l'objet d'une négociation directe entre la pharmacie de l'établissement de santé et le laboratoire. Hors ce cas particulier, la logique est celle d'un prix dit «administré ». II revient à l'źtat de fixer le montant auquel le produit peut être vendu et remboursé, au terme d'une négociation très cadrée à la fois par la Loi et par des accords conventionnels. Cette négociation est conduite par une instance interministérielle, le Comité économique des produits de santé (CEPS), sur la base des évaluations successives qui jalonnent le parcours réglementaire (le SMR, l'ASMR, l'efficience médico-économique) et des prix pratiqués dans les pays de référence. Point important, les industriels n'ont pas à justifier des coûts qu'ils supportent ou ont supporté pour argumenter le prix qu'ils demandent. Ils n'ont cette obligation ni en France, ni dans d'autres pays. Pour les médicaments particulièrement innovants et qui ne possèdent aucune alternative thérapeutique, les négociations de prix sont excessivement compliquées et souvent très longues, avec des rapports de force parfois assez disproportionnés. Les négociations de prix et les accords commerciaux conclus sont toujours secrets. Pour tous les médicaments très coûteux, le prix facial c'est-à-dire le prix public facturé, payé par l'hôpital et remboursé par l'Assurance maladie, s'avère de plus en plus souvent supérieur voire très supérieur au prix final réel une fois le système des remises de fin d'année mis en $œ u v r e$. Ces remises secrètes englobent notamment les accords de « caping », systématique au-delà d'un coût de traitement de 50 000 euros par patient et par an. Un tel accord consiste à se mettre d'accord sur un prix facial. Le CEPS convient d'acheter à ce prix

\section{3 sites institutionnels pour en savoir plus}

- L'Agence nationale de sécurité des médicaments et des produits de santé (ANSM) : https://www.ansm. sante.fr

- La Haute autorité de santé (HAS) : http://www. has-sante.fr

- L’Agence européenne des médicaments (EMA) https://europa.eu

public le médicament pour $X$ patients, $X$ étant toujours très inférieur au nombre de patients qui seront effectivement traités. De son côté, le laboratoire s'engage à traiter tous les patients qui médicalement en auront besoin. En fin d'année, le laboratoire rembourse tout ce qu'il a facturé au-delà de ce nombre $X$. C'est ainsi que, pour tous nos médicaments innovants, le prix payé par l'établissement de santé pour acheter le médicament, et qui lui est remboursé par l'Assurance maladie, peut se montrer très supérieur au prix réel, qui est protégé par le secret des affaires. Personne ne le connaît en dehors du CEPS et de l'industriel, ce qui complique la compréhension des données connues sur le prix de tel ou tel médicament. A son arrivée dans les établissements de santé, le médicament doit encore franchir d'autres étapes, réglementaires ou non. Délivrer un traitement innovant génère en effet une série de coûts associés qu'ils soient humains, techniques, organisationnels, de suivi, de registres... Ils ne sont pas pris en compte par les hôpitaux via des mécanismes aussi automatiques que ceux appliqués à l'achat du médicament, ce qui peut les mettre en difficulté. Certains établissements s'organisent pour faire face à ces coûts associés. Ailleurs, des retards ou des blocages sont introduits par la Commission du médicament et des dispositifs médicaux stériles (COMEDIMS) de l'hôpital. Elle donne son avis sur le rôle du médicament, alors même qu'une décision nationale a été prise à ce sujet et que, normalement, elle devrait s'imposer à tous. Mais dans la réalité, en attendant qu'elle se réunisse, parfois les patients ne peuvent pas commencer à être traités et ce retard est difficilement acceptable. II arrive enfin que des craintes financières, sans aucun fondement au regard de la réglementation, fassent hésiter certains hôpitaux. «Le médicament est trop cher. II ne sera pas remboursé... ». Encore une fois un produit coûteux, qu'il bénéficie d'une ATU, d'une post-ATU ou soit inscrit sur la liste en sus, ne pèse pas sur les finances d'un établissement puisqu'il se fait rembourser intégralement ses achats, à la boîte près. 


\section{Mutualiser les connaissances pour mieux anticiper}

Sur ce point comme sur de nombreux autres, la complexe et mouvante réglementation reste mal connue ou mal interprétée par certains acteurs locaux, a fortiori sur le champ des médicaments vraiment innovants. Dans ce contexte, le partage et la circulation d'informations réglementaires validées au sein la filière de santé des maladies rares neuromusculaires Filnemus parait vivement souhaitable. Le plan national maladies rares III, annoncé au début du mois de juillet 2018, prévoit la création d'un observatoire des traitements dans chaque filière de santé maladies rares (action 4.2). Ce pourrait être un outil pour échanger ces informations réglementaires et pour évaluer les conséquences de la mise sur le marché des nouveaux médicaments. Espérons que Filnemus puisse être suffisamment précurseur en ce domaine et mette en place le plus vite possible cette instance. Nous pourrons alors travailler collectivement à l'arrivée des thérapies innovantes.

The long path (s) of placing medicines on the market

\section{LIENS D'INTÉRÊT}

L'auteur déclare n'avoir aucun lien d'intérêt concernant les données publiées dans cet article.

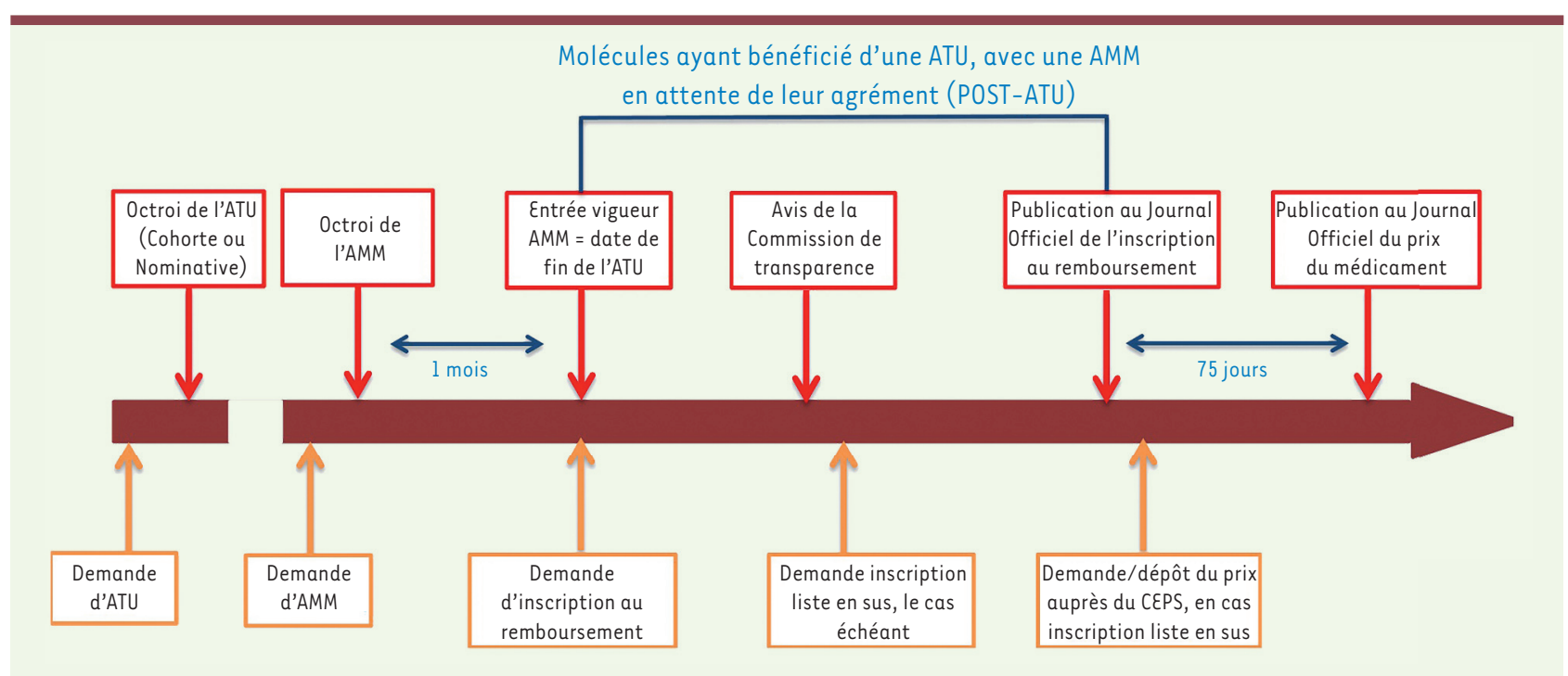

Figure 1. Le circuit des médicaments bénéficiant d'une ATU (source : Comptes de la Sécurité sociale, rapport de septembre 2017).

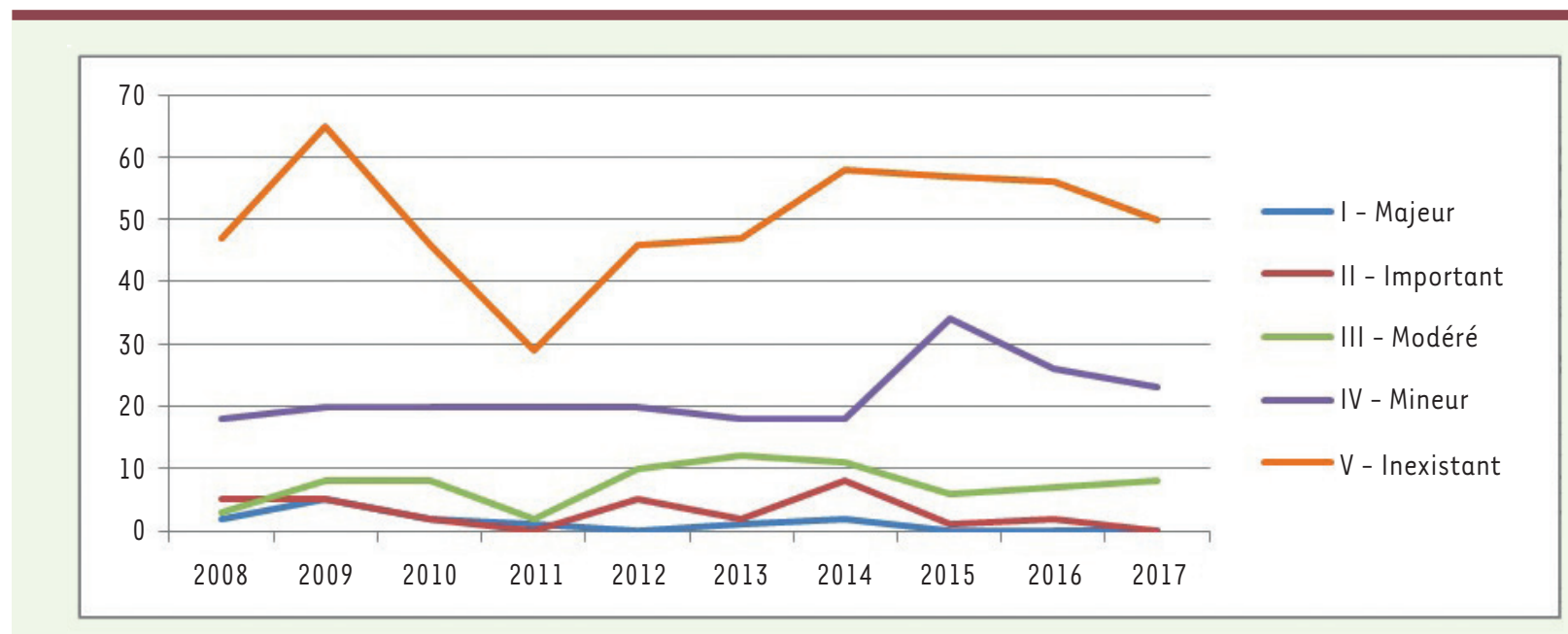

Figure 2. Évolution de la répartition des ASMR attribuées par la HAS (demandes de première inscription ou d'inscription dans une nouvelle indication) (source: HAS. Ces données ne portent pas sur les demandes d'avis ayant fait l'objet d'une procédure simplifiée). 